\title{
XI. Ausblick: Später Klimawandel in der japanischen Erinnerungskultur und "Amerikanisierung des Holocaust“ in der Bundesrepublik
}

Markierten die 1960er Jahre für die Bundesrepublik Deutschland eine Phase tiefgreifenden erinnerungskulturellen Wandels, so änderte sich das Klima in Japan erst im Gefolge eines großen Schulbuchstreits 1982 spürbarer. Allerdings hatten die von dem Professor an der Pädagogischen Hochschule in Tokio, Ienaga, 1965 bzw. 1967 eingereichten Klagen gegen den Staat bzw. das Erziehungsministerium u.a. wegen der Verletzung der verfassungsmäBig garantierten Meinungsfreiheit durch die staatliche Lehrbuchkontrolle 1970 und 1974 zu erstinstanzlichen (Teil-)Siegen Ienagas geführt ${ }^{1}$. Dies trug eine Zeit lang zur "Liberalisierung der Geschichtsdarstellung"2 in Schulbüchern bei und machte etwa die Darstellung der Eroberung von Nanking seit 1974 wieder möglich. Nicht nur in der rechtsgerichteten Publizistik, sondern auch in der regierenden LDP formierte sich aber bald Widerstand gegen „Nanking-Lüge“ und „tendenziöse Geschichtsschreibung“ - bis hin zur Gründung einer Art Task force der LDP gegen die Präsenz von Nanking in den Schulbüchern ${ }^{3}$.

Zunächst ähnlich wie bei den periodisch aufgeflackerten Kontroversen um die Praxis der Lehrmittelzulassung ging es beim großen Schulbuchstreit $1982 \mathrm{im}$ Kern um den von Japan in China geführten Krieg und vor allem auch um das Massaker von Nanking. Von „Invasion“ oder „Aggression“ zu reden, wie dies in einigen der diesmal zur Prüfung anstehenden Geschichtsbücher für die Oberschulen getan wurde, widersprach nach wie vor der Auffassung des Monbushô (Erziehungsministerium). Die Behörde wollte das Ereignis von Nanking in die Fußnoten verbannt wissen. Zudem verlangte sie Erklärungen, die das Vorgehen der japanischen Soldaten tendenziell entschuldigten. Auch die Kolonialpolitik in Korea sollte nach Ansicht der Tokioter Behörde in einem weniger schlechten Licht erscheinen, als dies in einigen Manuskripten der Fall war ${ }^{4}$. Nach wie vor also verstand das kon-

1 Petersen, Die Schulbuchprozesse, S. 64 f., 73.

2 Ishida, Das Massaker von Nanking, S. 238.

3 Ebd., S. 239.

4 Fuhrt, Erzwungene Reuc, S. 80-90. 
servativ beherrschte Ministerium Schulbuchgestaltung in erster Linie als Beitrag zur Systemstabilisierung und lehnte eine „negative“ Darstellung der Rolle Japans in den Aggressionskriegen ab5 .

$\mathrm{Da}$ diese Position hinlänglich bekannt und ausdiskutiert war, fanden die Eingriffe des Erziehungsministeriums in der japanischen Öffentlichkeit zunächst wenig Beachtung. Ganz anders aber als früher fiel die Reaktion in den Nachbarländern aus. Nach einem kritischen Artikel in dem chinesischen KP-Organ Renmin Ribao wurde dem japanischen Gesandten im chinesischen Außenministerium am 26. Juli 1982 eine förmliche Protestnote überreicht und deutlich gemacht, daß die vom Tokioter Ministerium vorgenommenen „Verfälschungen ... eindeutig eine Verzerrung historischer Tatsachen“ darstellten, die „in keiner Weise gebilligt"6 werden könnten. Pekings Entschlossenheit, den Verstoß gegen den Geist des Friedens- und Freundschaftsvertrags zwischen Japan und China nicht hinzunehmen, wurde durch die Ausladung des Erziehungsministers bekräftigt, der im September eigentlich nach China hatte reisen sollen.

Die Militärregierung in Südkorea, erst drei Jahre vorher durch einen blutigen Putsch an die Macht gelangt und international noch ziemlich isoliert, taktierte in der Hoffnung auf einen seit 1981 verhandelten japanischen Milliardenkredit zunächst zurückhaltender, sie geriet aber mehr und mehr unter den Druck der Japan-kritischen Presse und der auf den Straßen demonstrierenden Veteranenverbände. Die teils extremen antijapanischen Emotionen gipfelten in Steinwürfen auf das japanische Konsulat oder Boykottaktionen einheimischer Taxifahrer gegen japanische Touristen. So sah sich die Regierung in Seoul veranlaßt, einige Ventile zu öffnen und im August 1982 mittels einer diplomatischen Protestnote eine Änderung der umstrittenen Geschichtsbuchpassagen von Tokio zu fordern. Auch Staatspräsident Chun Doo-Hwan wies in seiner Rede zum Nationalfeiertag am 15. August die Darstellung des japanischen Erziehungsministeriums mit Nachdruck zurück, woraufhin sich die Lage in Südkorea selbst wieder etwas entspannte. $\mathrm{Da}$ aber in anderen Ländern Südostasiens, allen voran in Singapur mit seiner großen chinesischen Bevölkerungsgruppe, in der Öffentlichkeit ebenfalls massive Vorwürfe gegen die unzulängliche japanische „Vergangenheitsbewältigung" laut wurden, ja schwerer wiegend noch, Tokios militärischer Expansionsdrang von einst mit seiner Wirtschaftspolitik von heute argumentativ verknüpft wurde ${ }^{7}$, sah sich die japanische Regierung mit der bisher wohl schwierigsten, historisch bedingten außenpolitischen Problemkonstellation seit 1945 konfrontiert. 
Nachdem Tokio dem Ausland gegenüber jahrzehntelang mit einer Mischung halbherzig wirkender Erklärungen des Bedauerns und wirtschaftlicher Kooperationsbereitschaft ausgekommen war, während im Inneren gleichzeitig affirmative Rückschau auf die eigene Geschichte betrieben wurde ${ }^{8}$, stand der Regierung nun kein geeignetes Instrumentarium zur Krisenbewältigung zu Gebote. Vor allem im Erziehungsministerium fehlte es an Verständnis dafür, daß von der schönfärberischen Darstellung der dunkelsten zeitgeschichtlichen Kapitel auch die Gefühle und Interessen jener Nachbarvölker berührt waren, die japanische Besatzung und Kolonialherrschaft erfahren hatten. Aus Furcht, durch nachträgliche Revisionen der vorgenommenen Schulbuchkorrekturen einen Präzedenzfall zu Gunsten der traditionell vergangenheitskritischen Lehrergewerkschaft zu schaffen, bemühte sich das Monbushô statt dessen verstärkt, seine Politik im In- und Ausland zu rechtfertigen. Da China- und Südkorea-freundliche Gruppen der LDP aber vor allem die außen- und wirtschaftspolitischen Weiterungen des Problems sahen, herrschte im Regierungslager insgesamt Uneinigkeit über das weitere Verfahren, so daß Ministerpräsident Suzuki Zenko wochenlang dilatorisch agierte.

Erst als im August 1982 die Gefahr offensichtlich geworden war, daß ohne eine Beilegung des Konflikts der für September geplante China-Besuch Suzukis anläßlich des zehnten Jahrestages des Normalisierungsvertrages zumindest schwer belastet werden würde, den der Regierungschef eigentlich zur Selbstdarstellung vor seiner Wiederwahl als LDP-Vorsitzender auf dem Parteitag im Oktober nutzen wollte, gab Suzuki in einer öffentlichen Erklärung am 23. August die Zusicherung, eine Korrektur der umstrittenen Schulbuchpassagen einzuleiten. Die Konkretisierung neuer Richtlinien für die Schulbuchzulassung wurde allerdings dem Erziehungsministerium anvertraut. Daß die Position des Ministerpräsidenten nicht etwa aus einem veränderten historischen Bewußtsein resultierte, sondern aus taktischen Überlegungen außenpolitischer Art, erhellte auch seine Antwort auf die Frage eines Journalisten, ob er für die militärischen Aktionen Japans vor 1945 den Ausdruck „Invasion“ oder „Vorrücken“ für angemessen halte: „Die Bewertung der Taten unseres Landes vor dem Krieg sollte dem Urteil der Historiker späterer Generationen vorbehalten bleiben“, sagte Suzuki, aber es sei „eine Tatsache, daß es international, darunter auch in China, die strenge Bewertung, Beurteilung oder Einschätzung gibt, es sei eine Invasion gewesen. Dies müssen wir als Regierung hinreichend berücksichtigen"9.

Seoul und Peking reagierten zwar mit Enttäuschung auf die eher beschwichtigende als nachgiebige Haltung des Kabinetts Suzuki, doch daß beide Regierungen im Herbst 1982 die Angelegenheit für beendet erklärten, 
verwies auf den entscheidenden machtpolitischen Hintergrund dieser bisher größten Kontroverse um die japanische „Vergangenheitsbewältigung“: Die Kräfteverhältnisse in Ostasien hatten sich seit den 1960er Jahren, als Tokio bei seiner ablehnenden Haltung gegenüber koreanischen Wiedergutmachungsforderungen noch ganz auf die USA bauen konnte, zwar erheblich verschoben. Trotz dieses höher gewordenen Außendrucks kam der japanischen Regierung aber immer noch zugute, daß vor allem die Reformkräfte in China das Interesse an wirtschaftlicher Zusammenarbeit letztlich höher bewerteten als Fragen historischer Moral ${ }^{10}$.

Wenn der Schulbuchstreit von 1982 dennoch zumindest als kleiner Paradigmenwechsel in der japanischen „Vergangenheitsbewältigung“ interpretiert werden kann, so vor allem deshalb, weil Meinungsumfragen nunmehr belegten, daß die Stimmung in der Bevölkerung, gerade bei der jüngeren Generation, mehrheitlich zur Korrektur der umstrittenen Bücher neigte. Ein ähnliches Bild zeigte sich bald auch bei der Haltung in der Frage der „Wiedergutmachung“. Während eine Verpflichtung der japanischen Regierung zur Entschädigung von Kriegsopfern in einer Umfrage (1993) von einer relativen Mehrheit (mehr als 40 Prozent) der über Fünfzigjährigen verneint wurde, erreichte die Zahl der Befürworter unter den Zwanzig- bis Dreißigjährigen fast 70 Prozent ${ }^{11}$. Die über Jahrzehnte nahezu eingefroren wirkende Vorstellung, japanisches Volk und Tennô seien Opfer einer Militärclique geworden, der die Rolle des Übeltäters allein zufalle - diese „heroische Erzählung“ (C. Gluck) wurde jetzt doch zunehmend von einer nach 1945 geborenen Generation in Frage gestellt, die sich für die Frage der Kriegsverantwortung zumindest mehr zu interessieren begann, als dies unter den Älteren der Fall war ${ }^{12}$.

Auch die Rolle der Medien hatte sich im Vergleich zu den ersten Jahrzehnten nach 1945 erheblich gewandelt, weil es reißerisch aufgemachten Wochenblättern, alternativen und ausländischen Zeitungen, deren Journalisten nicht zur In-Group der nationalen Presseclubs gehörten, mehr und mehr gelang, tabuisierte Themen auf die Agenda zu bringen und die von Chalmers Johnson als „Kopfkartelle“ bezeichneten unsichtbaren Mauern zu durchbrechen ${ }^{13}$. Die Zeitungen verurteilten, wenn auch erst angesichts des wachsenden Außendrucks, nicht nur klar das Taktieren der Regierung,

10 Vgl. Miyaoka, Foreign Pressure and the Japanese Policymaking Process, sowie Fuhrt, Erzwungene Reuc, S. 109, 137.

11 Vgl. Fuhrt, Erzwungene Reue, S. 126f., S. 182.

12 Die Umfrage, die Asabi Shimbun 1993 durchgeführt hatte, zeigte nämlich andererseits ein hohes $\mathrm{Maß}$ an Desinteresse der jüngeren Generation gegenüber der Entschädigungsfrage. Fuhrt, Erzwungene Reue, S. 182. Vgl. auch Gluck, The Past in the Present, S. 344; HijiyaKirschnereit, „Kriegsschuld, Nachkriegsschuld“, S. 344; Mishima, Generationswechsel und Erinnerungskulturen in Japan.

13 Vgl. Hijiya-Kirschnereit, „Kriegsschuld, Nachkriegsschuld“, S. 343 f.; Pharr/Krauss, Media and Politics in Japan, S. 19-43. 
sondern sie begannen auch verstärkt, eine kontrollierende Funktion auszuüben, indem sie den immer zahlreicher werdenden Kritikern der Regierung ein breites Forum boten. In der Folge des Schulbuchstreits gründeten sich neue Gruppen von Eltern, Pädagogen, Schulbuchautoren und Intellektuellen, die sich der älteren Kritik von Lehrergewerkschaften, Historikerverbänden, Friedensgruppen und christlichen Gemeinschaften anschlossen und eine intensivere „Aufarbeitung“ der japanischen Kriegspolitik anmahnten $^{14}$. Auch die sozialistische Oppositionspartei entdeckte das Thema „Vergangenheitsbewältigung“ als Chance, sich gegen die herrschenden Konservativen zu profilieren.

Weiter begünstigt wurde die intensivere Beschäftigung mit der Kriegsvergangenheit durch den Tod Kaiser Hirohitos am 7. Januar 1989. Nicht nur weil das Motiv der Rücksichtnahme auf den ebenso verehrten wie historisch verstrickten Tennô entfiel, sondern auch weil viele Japaner zur Kenntnis nehmen mußten, daß die internationale Presse kritische Nachrufe auf Hirohito, vor allem wegen seiner Rolle beim Überfall auf China 1931 und während des Pazifisch-Ostasiatischen Krieges, publizierte. Das niederländische Parlament sah sich gar veranlaßt, gegen die Teilnahme von Königin Beatrix am Begräbnis zu intervenieren, weil es den Kaiser selbst für die Greueltaten in Niederländisch Ostindien (Indonesien) verantwortlich machte ${ }^{15}$. In Japan hatte man sich also nach dem Ableben Hirohitos abermals - wie schon 1982 - der Einsicht zu stellen, daß die anderen Völker die militaristische Vergangenheit Japans nicht einfach vergessen hatten ${ }^{16}$.

So wurde in China der als "direkte Antwort auf die Schulbuchrevision“ 1983 begonnene Bau eines 25000 Quadratmeter großen Erinnerungskomplexes für die - wie es auf einer Gedenkmauer heißt - „300000“ Opfer von Nanking anläßlich des 40. Jahrestags der japanischen Kapitulation am 15. August 1985 vollendet ${ }^{17}$. Dabei handelte es sich gleichsam um eine Gegenveranstaltung zu Nakasones offiziellem Besuch des Yasukuni-Schreins am gleichen Tag, der zudem in den großen chinesischen Städten von öffentlichen Protestdemonstrationen begleitet wurde ${ }^{18}$. In anderen ostasiatischen Nachbarländern Japans bewog die politische Liberalisierung viele der dort noch lebenden Kriegsopfer, vor allem die lange schweigenden „Trost-

14 Fuhrt, Erzwungene Reue, S. 105, 132, 139. Der kurz nach Einreichung der ersten Klage Ienagas gegründete Nationale Verband zur Unterstützung der Schulbuchprozesse erlebtc in den 1980er Jahren ein sprunghaftes Wachstum der Mitgliederzahlen und umfaßte 1992 etwa 25000 Einzelmitglieder sowie 2100 Gruppenmitglieder, darunter vor allem Lehrer- und Verlagsgewerkschaften. Petersen, Die Schulbuchprozesse, S. $77 \mathrm{f}$.

15 Coulmas, Das Land der rituellen Harmonie, S. 65.

16 Murakami, Bleibt Deutschland weiterhin Japans Modell, S. 4; Sato (Japan und der Zweite Weltkrieg, S. 125) vertritt demgegenüber die These, der "Tod einer einzigen Person“ habe eine „neue Ära“ im Umgang mit der nationalen Geschichte eingeleitet.

17 Yang, The Malleable and the Contested, S. 69.

18 Hardacre, Shintô and the State, S. 151. 
frauen“, Anfang der 1990er Jahre endlich dazu, Entschädigungsansprüche gegen Tokio geltend zu machen. Unterstützt von einer engagierten Gruppe sozialistischer Abgeordneter, Bürgerrechtsgruppen, Anwaltsvereinigungen und großen Teilen der japanischen Medien gelang es ihnen zumindest, die anfängliche Strategie der LDP-Regierung zu durchkreuzen, die jede staatliche Beteiligung am System der Zwangsprostitution während des Zweiten Weltkriegs kategorisch abstritt. Doch obwohl die Sozialisten nach dem Ende der LDP-Alleinherrschaft ab 1994 sogar den Ministerpräsidenten in einer Dreiparteienkoalition stellten, vermochten sie sich mit ihrem Konzept der staatlichen Beteiligung an einer Stiftung für alle Opfer des Krieges und der Kolonialherrschaft gegenüber konservativem Regierungspartner und Beamtenapparat nicht durchzusetzen. Mit der „Asiatischen Friedensstiftung des Volkes für Frauen“ kam 1995 schließlich nur ein privater Fonds zustande ${ }^{19}$.

Das konservative Beharren auf einem nationalapologetischen Geschichtsbild, das sich auch in einer „unwürdigen Debatte“ um die Resolution des Parlaments zum 50. Jahrestag des Kriegsendes niederschlug 20 , hatte bereits in den Vorjahren mehrfach zu politischen Skandalen bis hin zum Ministerrücktritt (aus allerdings vor allem außenpolitischen Rücksichten) geführt, als etwa der ehemalige Leiter der Geheimpolizei, Okuno Seisuke, 1988 bei einem Besuch des Yasukuni-Schreins sein Unverständnis darüber äußerte, daß Japan des Militarismus und der Aggression bezichtigt würde, wo doch die weiße Rasse Asien zur Kolonie gemacht hätte ${ }^{21}$. Bereits 1986 war Erziehungsminister Fujio Masayuki entlassen worden, nachdem er die Zahl der Opfer von Nanking in Zweifel gezogen hatte22, was im Mai 1994 Justizminister Nagano Shigeto nicht an der Behauptung hinderte, das Massaker von Nanking sei eine Erfindung westlicher Journalisten ${ }^{23}$. Ereignisse dieser Art machten wie schon der Verlauf der Schulbuchdebatte 1982 deutlich, daß sich die zunehmend kritische Haltung der Medien nicht einfach zu einem neuen politischen Konsens in Fragen mit zeitgeschichtlichem Bezug auswuchs, sondern nationalkonservative Politiker, Publizisten aber auch Wissenschaftler sich unvermindert vehement gegen die, wie sie es sahen, Verunglimpfung der japanischen Vergangenheit durch Thematisierung der Kriegsverbrechen zur Wehr setzten und dem den Wert der inneren Geschlossen-

19 Fuhrt, Erzwungene Reue, S. 156, 162 ff., $192 \mathrm{f}$. Vertiefend Seraphim, Der Zweite Weltkrieg im öffentlichen Gedächtnis Japans.

20 Kritische Bcobachter hatten einmal mehr zu monieren, daß in einem offiziellen Papier zur jüngsten Vergangenheit nur von "aggressionsähnlichen Handlungen“ Japans gesprochen und entschuldigend hinzugefügt wurde, nicht nur Japan, sondern alle Großmächte hätten sich durch „viele Akte von Kolonialherrschaft und Aggression in der Weltgeschichte“ schuldig gemacht. Schwentker, Täter oder Opfer?, S. 162.

21 Chang, Die Vergewaltigung von Nanking, S. 210.

22 Glaubitz, Japan und China, S. 203.

23 Schwentker, Täter oder Opfer, S. 143. 
heit und positiven Identität der Nation entgegensetzten. Die heutigen Japaner dürften in der Schule nicht als "Kinder von Verbrechern“ behandelt werden, und auch die Medien, so ihr Appell, dürften aus Achtung vor dem Vaterland nicht immer wieder "die angeblich vor 50 Jahren begangenen Greueltaten der Vorfahren in sensationeller Aufmachung“ anklagen ${ }^{24}$. Schon 1984 wurde wieder ein neues Geschichtsbuch zugelassen, „dessen Grundton ,erzkonservativ“ war" 25 .

Überdies hat der Tokioter Erziehungswissenschaftler Fujioka Nobukatsu, besessen von der abwegigen Vorstellung einer erinnerungskulturellen Hegemonie der Linken, in den letzten Jahren „Geschichten, von denen die Schulbücher schweigen" veröffentlicht und die historischen Heldentaten der Japaner im Zweiten Weltkrieg in über 800000 Exemplaren verkauft. Daß der ultrarechte Pädagogikprofessor Fujioka keineswegs ein Außenseiter ist, erhellt schon aus der öffentlichen Unterstützung seiner Thesen durch 62 Abgeordnete der LDP sowie durch fast ebenso viele Sympathisanten im Lager der konservativen Neuen Fortschrittspartei, die noch vor wenigen Jahren als Hoffnungsträger einer liberalen Erneuerung Japans gehandelt wurde. Damit steht etwa ein Fünftel der Parlamentarier hinter Fujioka und gegen die zaghaften Bemühungen einiger Premierminister um einen sensibleren Umgang mit der dunklen Vergangenheit ${ }^{26}$. Von einem gesellschaftlichen Konsens bezüglich der Bewertung des Ultranationalismus, ja auch nur des Charakters der japanischen Militäraktionen als „Angriffskrieg“ gegen seine asiatischen Nachbarn, wie ihn die Premiers Hosokawa und Murayama am 10. August 1993 bzw. am 15. August 1996 eingestanden²7, ist Japan aufs Ganze gesehen weit entfernt geblieben.

Der erinnerungskulturelle Paradigmenwechsel im Japan der 1980er Jahre griff jedenfalls viel weniger tief als in der Bundesrepublik zwei Dekaden zuvor, nicht zuletzt, weil er von einer viel niedrigeren Ebene auszugehen gehabt hatte. Im Grunde genommen trat die japanische „Vergangenheitsbewältigung" erst jetzt in eine politisch-pragmatische Phase ein, wie sie - cum grano salis - bereits nahezu die ganze Ära Adenauer gekennzeichnet hatte, ohne jedoch auch nur annähernd den hohen moralpolitischen Grat zu erreichen, auf dem die Bundesrepublik seit den 1960er Jahren immer weiter vorankam. Versucht man, einen Überblick über diese (west-)deutsche Entwicklung zu gewinnen, so lassen sich positive wie negative Veränderungen gegenüber dem Umgang mit der NS-Erblast in der Ära Adenauer feststellen.

Erfreulich waren zunächst die Impulse, die von der Herausforderung durch die „68er" auf die deutsche Geschichtswissenschaft ausgingen. Sie

24 Fuhrt, Erzwungene Reue, S. 123 (Zitat), 138.

25 Ishida, Das Massaker von Nanking, S. 239.

26 Mishima, Generationswechsel und Erinnerungskulturen, S. $356 \mathrm{f}$.; Lourdes Martínez, Zwangsprostitution und Entschädigung, S. 38.

27 Yagyû, Der Yasukuni-Schrein, S. 246. 
schlugen sich in neuen Untersuchungen zum Verhältnis von Politik, Wirtschaft und Gesellschaft in der Zeit des Dritten Reiches oder zum System der Konzentrationslager nieder sowie bald auch in einem verstärkten Interesse zahlreich sich gründender „Werkstätten“ an der Alltags- und Lokalhistorie des Dritten Reiches oder in der Einrichtung neuer bzw. Erweiterung bestehender KZ-Gedenkstätten zwischen Hamburg/Neuengamme (1981) und dem Oberen Kuhberg bei Ulm (1985). Auch Städte wie Nürnberg oder Berlin wurden jetzt aktiver, die in der Zeit des Nationalsozialismus eine exponierte Rolle gespielt, nach 1945 aber trotz ihrer meist sozialdemokratischen Ratsmehrheiten wohl aufgrund der spezifischen „Volksnähe“ der Kommunalpolitik diese Vergangenheit jahrzehntelang nicht gerade intensiv „aufgearbeitet" hatten.

Zu den Ursachen für diese neuen Entwicklungsschübe der bundesdeutschen Erinnerungskultur zählten nicht nur moralpolitische Impulse von innen, sondern einmal mehr starke Einflüsse von außen. Denn eine Initialfunktion für das vermehrte Engagement vor allem auch einer jüngeren $\mathrm{Ge}$ neration, die sich die "Schuldfrage“ nicht mehr selbst stellen mußte, sondern sie an Väter und Großväter richtete, hatte der emotional erschütternde US-Fernsehfilm „Holocaust“, den Ende Januar 1979 auch über 16 Millionen Deutsche sahen. Erst in der Folgezeit wurde der Begriff „Holocaust“ und dann auch "Auschwitz“ zum Synonym des Grauens, das Deutsche während der Hitler-Zeit über die Welt gebracht hatten ${ }^{28}$. Die Entstehung und Wirkung des Films in den USA - und mittelbar dann auch in Deutschland - hatte sich einem Klima verdankt, das mit der These von der „Amerikanisierung des Holocaust" beschrieben worden ist. Danach wurde der Holocaust im Kontext der israelisch-arabischen Kriege 1967 und 1973 zu einem jüdisch-amerikanischen Deutungsmuster, das sich auf den Nahost-Konflikt übertragen ließ und die israelische Politik durch die Gleichsetzung von $\mathrm{Pa}$ lästinensern mit „Nazis“ vor Kritik abschirmte ${ }^{29}$. Die zum Bau des Holocaust Memorial Museum in Washington führende Initiative hatte 1978 - auf einer Feier zum 30. Jahrestag der israelischen Staatsgründung - tatsächlich US-Präsident Jimmy Carter ergriffen, nachdem der Verkauf von F-15 Kampfflugzeugen an Saudi-Arabien und Andeutungen über ein national home für die Palästinenser ihm seitens jüdischer Organisationen den Vorwurf der Äquidistanz zum arabischen und israelischen Lager eingetragen hatte ${ }^{30}$.

28 Winkler, Der lange Weg, S. 440.

29 Vgl. hierzu neben der erwähnten Studie von Novick, Nach dem Holocaust, S. 195-263: Berenbaum, The Americanization of the Holocaust; Junker, Die Amerikanisierung des Holocaust, sowie die Diskussion um Norman Finkelsteins Buch über „Die Holocaustindustrie“, in dem zentrale Thesen Novicks in provokativer und teils inakzeptabler Weise zugespitzt wurden. Piper, Gibt es wirklich eine Holocaust-Industrie?; Steinberger, Die Finkelstein-Debatte.

30 Vgl. Novick, Nach dem Holocaust, S. 278 f., der vor allem auch darauf verweist, daß „die Ju- 
Der Holocaust rückte jetzt rasch vom Rand in das Zentrum der politischen Kultur Amerikas vor, mit der Folge, daß es zwischenzeitlich in jeder größeren Stadt ein Holocaust-Museum gibt, aber auch Phänomene der „Trivialisierung und Vermarktung des, Shoah-Business" " zu beobachten sind. $\mathrm{Zu}$ den wesentlichen Bedingungen für die Möglichkeit dieser Entwicklung zählte es, daß die „Amerikanisierung des Holocaust“ als „ständige Konfrontation mit dem absolut Bösen“ den US-Bürgern die Chance bot, „das Böse zu externalisieren und zugleich die Notwendigkeit der eigenen Mission, der freiheitlich-demokratischen Sendung, zu erneuern“31. Parallel dazu setzte ein „olympischer Wettbewerb um die Goldmedaille für die größte Leidensgeschichte“ ein (P. Novick), in dem die amerikanischen Juden „so lange einen uneinholbaren Vorsprung “ behaupteten, wie sie ihr Land von der „Einzigartigkeit" und „Unvergleichlichkeit“ des Holocaust überzeugen konnten ${ }^{32}$.

Die für massenkulturelle Einflüsse aus den USA generell weit offene, „westernisierte“ deutsche Gesellschaft ${ }^{33}$ war auch für die „Amerikanisierung des Holocaust“ empfänglich. Allerdings bedeuteten diese Impulse keinen neuerlichen Paradigmenwechsel in der „Vergangenheitsbewältigung“. Vielmehr verstärkten sie nur noch die ohnehin wachsende Binnendynamik der bundesdeutschen Erinnerungskultur. Auf das Drängen der im Mentalitätswandel nach 1968 entstandenen grünen Partei ${ }^{34}$ war es etwa zurückzuführen, daß in den 1980er Jahren und vor allem dann nach dem Untergang des Sowjetblocks 1990 das Schicksal von Opfergruppen auf die politische Agenda in der Bundesrepublik kam, die bislang aus unterschiedlichen Gründen relativ wenig Aufmerksamkeit erfahren hatten, von den sogenannten „Asozialen“ bis zu den Zwangsarbeitern aus den osteuropäischen Ländern. Die Zwangsarbeiter waren zuerst dem Nationalsozialismus, dann aber dem Kalten Krieg zum Opfer gefallen, so daß im Sommer 2000, als der Bundestag nach mehreren vergeblichen Anläufen endlich mit großer Mehrheit cine „Stiftung Erinnerung, Verantwortung, Zukunft“ beschloß, von den über 20 Millionen Betroffenen nur noch etwa jeder zehnte am Leben war. Die eher symbolischen Entschädigungssummen betrugen zwischen 5000 und 15000 Mark $^{35}$.

den traditionell einen beträchtlichen Teil zum Budget für den Bundeswahlkampf der Demokraten beisteuerten" (S. 279), sowie Shafir, Die Rolle der Amerikanisch-Jüdischen Organisationen, S. 70.

31 Junker, Die Amerikanisierung des Holocaust.

32 Ebd.

33 Vgl. Doering-Manteuffel, Wie westlich sind die Deutschen?

34 Vgl. hierzu Assmann/Frevert, Geschichtsvergessenheit, S. $262 \mathrm{f}$.

35 Vgl. Reichel, Vergangenheitsbewältigung, S. $94 \mathrm{ff}$. Zur parteipolitischen Debatte um die Entschädigung von NS-Zwangsarbeitern in den 1980er Jahren aus "grüner" Sicht vgl. Saathoff, Die politischen Auseinandersetzungen, S. $55 \mathrm{ff}$. 
Ob wesentliche Ergebnisse der zweiten bundesdeutschen Bewältigungsphase auch ohne den moralpolitischen Paradigmenwechsel der 68er-Zeit einfach infolge der Überwindung des Ost-West-Konflikts erzielt worden wären, muß Spekulation bleiben. Evident sind einige gravierende Folgen für die politische und wissenschaftliche Kultur. So begann der „Faschismusverdacht" mancher "Betroffener" nun schon bei „rechten" Sozialdemokraten, was zu der kritischen Einschätzung auch von SPD-Politikern wie Klaus von Dohnanyi beigetragen haben mag, die Geschichte der NS-Zeit sei im politischen Tageskampf allzu oft „, in fragwürdiger Weise bemüht" worden ${ }^{36}$, etwa gegen Forderungen, türkischen Zuwandererkindern das Erlernen der deutschen Sprache zur Pflicht zu machen („rassistisch“, „Zwangsgermanisierung“37), oder gegen die Volkszählung, weil angeblich die Nationalsozialisten ohne entsprechende öffentliche Register nicht in der Lage gewesen wären, die jüdischen Bewohner aufzuspüren ${ }^{38}$. Obwohl es also eigentlich gerade zu den Folgen der NS-Katastrophe in (West-)Deutschland gehörte, daß Demokratie und Verfassungsstaat zum ersten Mal in der Geschichte der Nation „Gemeingut linker und rechter Demokraten geworden“ waren, konnte nun der Eindruck entstehen, „Vergangenheitsbeschwörung“ würde mehr und mehr in den Dienst einer „La démocracie c'est nous“-Attitüde ${ }^{39}$ gestellt.

In dem entstandenen gesellschaftlichen Klima führte der normale politische Vorgang der Ablösung der SPD-FDP-Regierung durch ein liberalkonservatives Kabinett 1982 mancherorts zu starker Besorgnis um den Fortbestand des zwischenzeitlich gewonnenen „kulturellen Hegemonieanspruchs" 40 . Hans Mommsen erkannte in den Plänen des CDU-Bundeskanzlers Helmut Kohl zur Errichtung historischer Museen in Berlin und Bonn, an dem namhafte Historiker wie Lothar Gall, Klaus Hildebrand, Horst Möller und Hans-Peter Schwarz beteiligt waren, den Versuch, den Deutschen zu neuem „Nationalstolz" zu verhelfen und den Nationalsozialismus zu relativieren ${ }^{41}$, obwohl etwa Horst Möller keinen Zweifel daran ließ, daß es bei der Suche nach der historischen Identität der Bundesrepublik „nicht um Verdrängung der NS-Epoche aus unserem Geschichtsbild“ gehe $^{42}$. Heftige Kritik zogen auch Ausführungen des CDU/CSU-Fraktionsvorsitzenden Alfred Dregger zur Bedeutung eines deutschen Patriotismus auf sich, weil sie bei manchen den Eindruck erweckten, mit der Wende in Bonn sei ein tragendes Element der politischen Kultur der Bundesrepu-

36 Dohnanyi, Hat uns Erinnerung das Richtige gelehrt?, S. 8.

37 Ebd., S. 11.

38 Ebd., S. 10.

39 So eine vorsichtig, in Frageform formulierte These Kielmanseggs, Lange Schatten, S. 94.

40 Hehl, Kampf um die Deutung, S. 425.

41 Mommsen, Neues Geschichtsbewußtsein, S. 175.

42 Allerdings könne diese Identität „heute nicht mehr nur" mit der Abkehr von der NS-Epoche begründet werden, sondern müsse auch positive Traditionen, die älter seien als der Nationalsozialismus, mit einbeziehen. Möller, Schuld und Verhängnis, S. 231, 234. 
blik allmählich aufgegeben worden: der Konsens, daß die Erinnerung an den Nationalsozialismus zu bewahren sei43.

Tatsächlich aber erreichte die von der Führung des westdeutschen Staates parteiübergreifend seit den Gründerjahren vorgenommene Distanzierung vom Dritten Reich gerade jetzt um den vierzigsten Jahrestag der Kapitulation Hitler-Deutschlands eine neue Stufe, als der christdemokratische Bundespräsident Richard von Weizsäcker in seiner Rede zum 8. Mai 1985 in aufrüttelnd klarer Sprache das Leiden der jüdischen Bürger im Dritten Reich und die Mitwisserschaft der Deutschen schilderte. Auch der erzwungene Rücktritt des Bundestagspräsidenten Philipp Jenninger nach einer Rede zum fünfzigsten Jahrestag der sogenannten „Reichskristallnacht“ am 10. November 1988, die zwar rhetorisch verunglückt, inhaltlich aber so untadelig war, daß sie Ignaz Bubis bei einer anderen Gelegenheit - und ohne irgendwelches Aufsehen zu erregen - selbst halten konnte, sprach nicht für eine nachlassende Erinnerung der bundesdeutschen Gesellschaft an den $\mathrm{Na}$ tionalsozialismus ${ }^{44}$. Vielmehr setzte sich gerade Mitte der 1980er Jahre, wenn vielleicht auch nicht - wie Heinrich August Winkler urteilt - „im allgemeinen Bewußtsein" ${ }^{45}$, so doch in einflußreichen Teilen der Gesellschaft endgültig die Auffassung durch, nicht nur den Verlust der Ostgebiete, sondern nun auch die Teilung Deutschlands selbst als Strafe und Sühne für die deutsche Schuld, für die Entfesselung des Zweiten Weltkriegs und für den Holocaust dauerhaft akzeptieren zu müssen ${ }^{46}$, d. h. nicht mehr nur realpolitisch als Unterpfand für das Gleichgewicht der beiden Blöcke und den Weltfrieden im Atomzeitalter. Daß man die apokalyptische Dimension des Holocaust durchaus sehen, es aber dennoch für moralisch unvertretbar halten konnte, allein die Deutschen in der DDR durch den dauerhaften Entzug elementarer Freiheitsrechte für die Verbrechen des Dritten Reiches büßen $\mathrm{zu}$ lassen, beschrieb eine Position wiedervereinigungspolitischen Idealismus', die jetzt als nicht mehr recht salonfähig, wenn nicht sogar „ewig gestrig“" galt. Auch der sogenannte Historikerstreit lieferte hierfür manches Anschauungsmaterial ${ }^{47}$.

43 Benz, Zum Umgang mit der nationalsozialistischen Vergangenheit, S. 56.

44 Dies zeigte sich nicht zuletzt auch in der erbitterten Kontroverse, die Bundeskanzler Kohl auslöste, als er gemeinsam mit Ronald Reagan 1985 den Soldatenfriedhof Bitburg besuchte, wo 2000 deutsche Soldaten, darunter auch 49 Angehörige der Waffen-SS begraben lagen, oder in der Debatte um Helmut Kohls Wendung von der "Gnade der späten Geburt", die teilweise „bewußt fehlinterpretiert" wurde. Hehl, Kampf um die Deutung, S. 426.

45 Vgl. Winkler, Der lange Weg, S. 439 f., Zitat S. 440.

46 Auschwitz, so der grüne Politiker Joseph Fischer wenige Tage nach dem Fall der Berliner Mauer, mache eine Selbstbestimmung der Deutschen auf Generationen hinaus unmöglich. Taz, 16. 11. 1989. 1989 wies im SPD-Parteivorstand der Historiker und Regierende Bürgermeister von Berlin, Walter Momper, Klaus von Dohnanyis Forderung nach entschlossener Vereinigungspolitik mit einem Generalverdikt über das Bismarck-Reich zurück. Dohnanyi, Erinnerung, S. 26.

47 Nach den fragwürdigen Thesen Ernst Noltes in der Frankfurter Allgemeinen Zeitung (v. 
Der enge Nexus zwischen Erinnerungskultur und Deutschlandpolitik blieb bis in die Tage der deutschen Revolution im Herbst 1989 hinein spürbar. So sah sich nach dem Fall der Berliner Mauer der SPD-Ehrenvorsitzende Willy Brandt veranlaßt, vor dem Bundesparteitag am 18. Dezember 1989 die Vorstellung zurückzuweisen, „noch so große Schuld einer Nation“ könne durch „willige Selbstbeschränkung oder gar Selbstverleugnung“ politisch gesühnt werden ${ }^{48}$. Sein politischer Enkel Oskar Lafontaine, für den die „Wurzeln [der Bundesrepublik]) in Auschwitz" 49 lagen, erweckte allerdings als Kanzlerkandidat im Herbst 1990 den Eindruck, dem vollzogenen Wiedervereinigungsprozeß nach wie vor ablehnend gegenüberzustehen.

Ausländische Deutschland-Kenner haben schon im Kontext der Nachrüstungsdebatte der frühen 1980er Jahre eine Fixierung der deutschen „Vergangenheitsbewältigung " auf Auschwitz registriert; dagegen bleibe die Problematik der westlichen Appeasement-Politik nach 1933 und des Verzichts auf einen Präventivkrieg gegen Hitler in der Erinnerungskultur der Bundesrepublik weitgehend ausgespart ${ }^{50}$. Nachdem der grüne Bundestagsabgeordnete Joseph Fischer 1983 die Logik der nuklearen Abschreckung in einen Zusammenhang mit der nach Auschwitz führenden „Systemlogik der Moderne" gebracht hatte, löste der CDU-Generalsekretär Heiner Geißler einen Sturm der Entrüstung aus, als er der „Friedensbewegung“ vorwarf, erst der Pazifismus und die Beschwichtigungspolitik der demokratischen Staaten gegenüber Hitler hätten Auschwitz möglich gemacht. Kanzler Kohl sprang Geißler bei, indem er im Parlament ausführlich aus dem Buch „The Gathering Storm" von Winston Churchill vorlas, der entschieden die Auffassung vertreten hatte, daß eine präventive Besetzung Hitler-Deutschlands 1938 die weitaus größere Katastrophe des Zweiten Weltkriegs und des Holocaust hätte verhindern können. Doch die folgende öffentliche Debatte dokumentierte, daß das von britischen und amerikanischen Liberalen immer noch sehr geschätzte Buch Churchills „im intellektuellen Leben Deutschlands nach dem Krieg nur eine sehr geringe Rolle gespielt" 51 hatte.

6. Juni 1986) zum „Klassenmord“ der Bolschewiki als logischem und faktischem Prius des „Rassenmords“ der Nationalsozialisten replizierte der Philosoph Jürgen Habermas nicht nur scharf auf den seines Erachtens von Nolte unternommenen Versuch, die Hypotheken der deutschen Vergangenheit abzuschütteln; er warf zudem weiteren eher konservativen $\mathrm{Hi}$ storikern vor, gleichsam im Dienste der Regierung Kohl das deutsche Nationalbewußtsein wiederbeleben zu wollen. Mittels der nationalapologetischen "Geschichtspolitik"Noltes, so fürchteten andere, sollten wohl auch die in jener Zeit verstärkt erhobenen Forderungen des FAZ-Mitherausgebers Joachim Fest nach einer Wiedervereinigung Deutschlands untermauert werden. Um die Wiederherstellung des Deutschen Reiches fordern zu können, müsse „die Geschichte in der Tat umgeschrieben werden“, dürfe das NS-Regime „nicht mehr als das erscheinen, was es war: das menschenfeindlichste der Geschichte ..." Winkler, Der lange Weg, S. 445.

48 Assmann/Frevert, Geschichtsvergessenheit, S. 65.

49 Lafontaine, Die Gesellschaft der Zukunft, S. 189.

50 Herf, Die Appeaser, sowie ders., Demokratie auf dem Prüfstand, S, $24 \mathrm{f}$.

51 Herf, Die Appeaser. 
Die seit den 1980er Jahren unverkennbare Fokussierung der deutschen Erinnerungskultur auf Auschwitz hat - über parteipolitische Grenzen hinweg - auch Kritiker gefunden, die es für riskant halten, ein bestimmtes $\mathrm{Ge}$ schichtsbild „zum Moralfundament einer Gesellschaft zu erklären“, und die an Jürgen Habermas' Diktum zweifeln, die Kulturnation der Deutschen habe erst "nach und durch Auschwitz" eine in Überzeugung verankerte Bindung an universalistische Verfassungsprinzipien ausgebildet. So richtete Dieter Langewiesche an Habermas' kategorische Ablehnung eines identifikatorischen Zugriffs auf die nationale Geschichte die Frage, ob die Verfassungen von 1849 und 1919 etwa nicht dazu taugten, in den „öffentlichen Gebrauch“ von Geschichte einzugehen ${ }^{52}$ ? Karl Heinz Bohrer hat anläßlich der Einrichtung der neuen Heidelberger Gadamer-Professur ebenfalls kritisiert, daß der Raum des deutschen Geschichtsbewußtseins „auf das Davor und Danach des Zivilisationsbruchs“ geschrumpft sei; nachdem der „moralisierende Umgang mit der Geschichte ... seit den sechziger Jahren einen triumphalen Erfolg erlebt“ habe, besitze Deutschland heute in Europa „das breiteste zeitgeschichtliche und das kürzeste historische Gedächtnis“"53. Auf eine weitere, internationale Kehrseite der Amerikanisierung des Holocaust hat der US-Germanist Mark M. Anderson verwiesen und bedauert, daß sich das Interesse an Deutschland in den USA auf „Hitler und den Holocaust“ weitestgehend reduziere ${ }^{54}$.

Gewiß wäre es problematisch, wenn die - wie Eckhard Fuhr es ausdrückte - „fast libidinösen Beziehungen zur Schuldgeschichte Deutschlands“ 55 in Teilen der Gesellschaft wirklich einem gleichsam „negativen Nationalismus“ in der Bundesrepublik Auftrieb gäben, einem „Gemeinschaftsgefühl mit pseudoreligiösen Zügen“ 56 , das die Idee des auserwählten Volkes durch Wendung ins Negative noch einmal bestätigte und den deutschen Sonderweg für alle Ewigkeit besiegelte. Diese Haltung des deutschen „Schuldstolzes“57 hat auch Aleida Assmann analysiert: „Kontaminiert mit dem absolut Bösen in ihrer Geschichte“ von den Befreiungskriegen über die

52 Antworten auf Jürgen Habermas, in: Frankfurter Allgemeine Zeitung, 18. Juni 1999.

53 Moralschmelze. Karl Heinz Bohrer liest: Attacke auf die Erinnerungskultur, in: Frankfurter Allgemeine Zeitung, 31. Mai 2001.

54 Anderson belegt dies mit Beispielen aus seinen New Yorker Lehrveranstaltungen. Vgl. Mark M. Anderson, Atlantisches Zerrbild, in: Die Zeit, 4. Oktober 2001. Die deutschen Nachbarländer hätten es sich, so kommentierte der niederländische Publizist Paul Scheffer den Sachverhalt, "mit einer gewissen Hingabe im Schatten der Untaten, die in deutschem Namen begangen wurden, bequem gemacht" - teilweise auch, um von schwarzen Blättern im Buch der eigenen Geschichte abzulenken. Paul Scheffer, Das Vertrauenskapital schwindet. Die Walser-Bubis-Kontroverse zeigt, daß die Zeit der Vormundschaft über Deutschland vorbei ist, in: Frankfurter Allgemeine Zeitung, 12. Dezember 1998, S. 11.

55 Eckhard Fuhr, Westen, was sonst?, in: Frankfurter Allgemeine Zeitung, 8. Juni 1994.

56 Winkler, Der lange Weg, S. 446.

57 Vgl. zu diesem Begriff die während einer Kontroverse um Martin Walser im Merkur publizierte Kritik von Cora Stephan, Schuldstolz. 
Reichsgründung bis zum Holocaust, so die dahinter steckende Vorstellung, hielten die Deutschen „einen Negativrekord gegenüber allen anderen $\mathrm{Na}$ tionen"58. Die Frage bleibt allerdings, welchen gesamtgesellschaftlichen Stellenwert diese extreme Ausbuchtung moralpolitisch geprägter Erinnerungskultur in der pluralistischen Demokratie Deutschlands tatsächlich erreicht hat. Wenn eine evangelische Landesbischöfin ein Vorwort zu einem Buch mit pädagogischen Anleitungen für den Holocaust-Unterricht im Kindergarten beisteuert, findet sie hierfür jedenfalls nicht nur Zustimmung59. Überdies bleibt abzuwarten, wie sich die „Rückkehr ... der deutschen Opfererinnerung "60 auswirken wird, die nach Günter Grass' Novelle über den Untergang der „Wilhelm Gustloff“ 61 oder Jörg Friedrichs Buch über den alliierten Bombenkrieg gegen Deutschland ${ }^{62}$ in letzter Zeit verstärkt zu beobachten ist ${ }^{63}$.

Wer die deutsche Entwicklung von den 1970er Jahren bis in die Gegenwart mit den Vorgängen in Japan vergleicht, wird nur mit Einschränkungen konstatieren können, die Formen der „Vergangenheitsbewältigung“ in beiden Ländern hätten sich - nach dem extremen Divergieren in den 1960er Jahren - wieder angenähert. Denn der - sehr stark durch wachsenden Außendruck bedingte - Aufholprozeß in Japan verlief derart ambivalent, blieb so sehr im Politisch-Pragmatischen stecken, daß es in der deutschen Erinnerungskultur wohl eines Rückschritts bedurft hätte, um wirklich von einer Annäherung zwischen beiden Ländern sprechen zu können. Tatsächlich aber gab es in der Bundesrepublik im Bereich der „Vergangenheitsbewältigung" keinen gesellschaftlichen Stillstand, sondern eine innere moralpolitische Eigendynamik, die zudem weiterhin durch äußere Impulse, jetzt durch die in den 1970er Jahren einsetzende "Amerikanisierung des Holocaust“, gespeist worden ist. Vielleicht kann es als Symbol dieser Entwicklung gelten, daß der Bau des zentralen Berliner Holocaust-Mahnmals mit dem brillanten Architekten Peter Eisenmann einem Angehörigen der jüdisch-amerikanischen Bevölkerungsgruppe anvertraut wurde ${ }^{64}$.

58 Assmann/Frevert, Geschichtsvergessenheit, S. 66.

59 Jäger, Das Leid, der Kitsch und das Geld.

60 Frevert, Geschichtsvergessenheit, S. 9.

61 Grass, Im Krebsgang.

62 Friedrich, Der Brand.

63 Frevert, Geschichtsvergessenheit, S. 10, betont allerdings auch, daß Bombenkrieg und Vertreibung in der deutschen Erinnerungsgeschichte vorher nicht stets vollständig tabuisiert waren. Offensichtlich ist aber doch - wie es die langjährige Warschau-Korrespondentin der Wochenzeitung Die Zeit, Helga Hirsch, ausgedrückt hat -, daß es „nach 1968 ... als politisch unkorrekt" galt, „über Deutsche als Opfer zu sprechen“. Hirsch, Flucht und Vertreibung, S. 25.

$64 \mathrm{Zu}$ den Mahnmalsentwürfen von Eisenmann und der Debatte um sie vgl. etwa Kirsch, Nationaler Mythos oder historische Trauer?, S. $288 \mathrm{ff}$. 